\title{
Application of suction-type cigarette drain in leak-prone hepatopancreatobiliary surgery
}

\author{
Shin Hwang, Dong-Hwan Jung, and Tae-Yong Ha
}

Department of Surgery, Asan Medical Center, University of Ulsan College of Medicine, Seoul, Korea

\begin{abstract}
Backgrounds/Aims: Penrose drain and Jackson-Pratt (JP) drain have benefits and drawbacks in terms of functional reliability of prolonged duration and easiness of bedside management. We have developed a suction-type cigarette drain (SCD) to compensate for the drawbacks. Methods: We have used SCDs in leak-prone or contaminated hepatopancreatobiliary surgeries since 2020. Herein, we describe key elements of our 20-year experience with the management of SCDs. Results: The SCD is available as two-piece and three-piece types. The two-piece SCD is a cigarette drain consisted of a silastic Penrose drain and Jackson-Pratt (JP) drain bearing multiple side holes. The three-piece SCD is a cigarette drain consisted of a silastic Penrose drain, internally located Nelaton catheter with multiple side-holes, and a connecting JP drain tube and bulb. These SCDs were inserted into the abdominal wall with a hemostat clamps and sealed with air-tight ties. Three sets of SCDs were usually inserted in more than 100 cases of patients who underwent pancreatoduodenectomy, and none of them underwent repeat surgery for postoperative pancreatic fistula. In more than 30 patients with extensive abdominal contamination, a single SCD was usually inserted into the high-risk point of fluid collection and none of them underwent repeat surgery to manage collection of abnormal fluid or abscess formation. Conclusions: Our experience suggests that SCD is highly reliable and acceptably convenient for clinical application in abdominal drainage. (Ann Hepatobiliary Pancreat Surg 2020;24:305-308)
\end{abstract}

Key Words: Penrose drain; Jackson-Pratt drain; Cigarette drain; Pancreatectomy; Abscess

\section{INTRODUCTION}

Commercially available intra-abdominal drains including open drains such as Penrose drain or closed suction drains such as Jackson-Pratt (JP) drain have benefits and drawbacks in terms of functional reliability of prolonged duration and ease of bedside management. To compensate for these drawbacks, we designed a suction-type cigarette drain (SCD) in early 2000, ${ }^{1}$ and have been using it for the past 20 years. SCD is a combination of cigarette and Jackson-Pratt drains, which facilitates maintenance of drain function for a prolonged duration and easy drain management. We herein describe our know-how to fabricate and manage SCDs based on our 20-year experience.

\section{METHODS TO FABRICATE AND INSTALL SUCTION-TYPE CIGARETTE DRAINS}

A two-piece SCD requires a silastic Penrose drain measuring $1 \mathrm{~cm}$ in diameter and $30 \mathrm{~cm}$ in length, and a JP drain with a $4.8 \mathrm{~mm}$-sized tube (Fig. 1). Multiple 1 $\mathrm{cm}$-sized semilunar side-holes were made using curved scissors to cut at the $15 \mathrm{~cm}$-long area from one end of the Penrose drain. The slit-shaped drainage portion of a JP drain tube is cut off and a metal trocar is also pulled out. Multiple small side-holes were also made by cutting with curved scissors at the $15 \mathrm{~cm}$-long area from one end of a JP drain tube. These two tubes are immersed in the saline for lubrication. The embossed end of a metal trocar was inserted into the side-hole portion of a silastic Penrose drain and the drain sheath was completely inserted

Received: July 25, 2020; Revised: July 26, 2020; Accepted: July 26, 2020

Corresponding author: Shin Hwang

Department of Surgery, Asan Medical Center, University of Ulsan College of Medicine, 88 Olympic-ro 43-gil, Songpa-gu, Seoul 05505, Korea Tel: +82-2-3010-3930, Fax: +82-2-3010-6701, E-mail: shwang@amc.seoul.kr

Copyright (C) 2020 by The Korean Association of Hepato-Biliary-Pancreatic Surgery

This is an Open Access article distributed under the terms of the Creative Commons Attribution Non-Commercial License (http://creativecommons.org/ licenses/by-nc/4.0) which permits unrestricted non-commercial use, distribution, and reproduction in any medium, provided the original work is properly cited. Annals of Hepato-Biliary-Pancreatic Surgery - pISSN: 2508-5778 - elSSN: 2508-5859 
over the trocar. The trocar-coupled Penrose drain tube was immersed in the saline again for further lubrication. The side-hole portion of the JP drain tube was connected to the embossed end of the metal trocar. The trocar was gently pulled out to insert the JP drain tube into the lumen of a silastic Penrose drain with matching side-holes, to make a cigarette drain (Fig. 1).

A $4 \mathrm{~mm}$-sized skin incision was made with a No. 15 surgical blade and a sharp hemostat clamp was inserted into the avascular layers of the abdominal wall. The overlapped end-portion of the cigarette drain without side-holes was firmly grasped by the clamp and pulled out to penetrate the abdominal wall with the cigarette drain. The overlapped end of the cigarette drain was tagged and sutured at the skin after inserting the side-hole portion adequately into the abdomen, and then the drain tube was tied firmly twice to prevent air leak. Finally, a suction

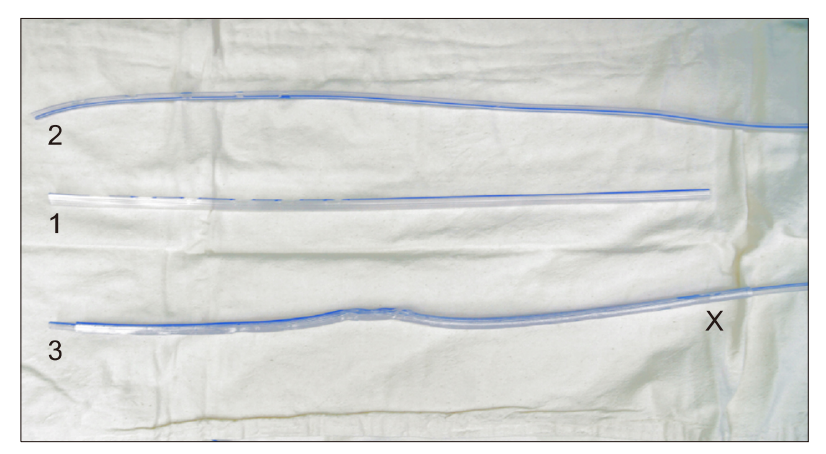

Fig. 1. Intraoperative photograph showing two-piece suction-type cigarette drain. Multiple $1 \mathrm{~cm}$-sized side-holes are made at the $15 \mathrm{~cm}$-long area from one end of a Penrose drain (1). Multiple small side-holes are made at the $15 \mathrm{~cm}$-long area from one end of the Jackson-Pratt (JP) drain tube (2). The JP drain tube is located within the lumen of the Penrose drain matching the side-hole portions, resulting in a cigarette drain (3). The overlapped end of the cigarette drain without side-holes $(\mathrm{X})$ is firmly held by a hemostat clamp and pulled out to penetrate the abdominal wall using the cigarette drain. bulb was attached, similar to the usual JP drain (Fig. 2). Continuous expansion of the bulb with air indicates air leak through the space between the Penrose and JP drain tubes, thus reinforcing the air-tight seal on the cigarette drain.

In addition to the two-piece SCD, a three-piece PCD had been used, which was our older method. It requires a silastic Penrose drain measuring $1 \mathrm{~cm}$ in diameter and 20-25 cm in length, a JP drain with a $4.8 \mathrm{~mm}$-sized tube, and a Nelaton catheter (Fig. 3A). It also requires a small plastic connector, which was pulled out of the intravenous infusion line. Multiple side-holes were made at the 10 $\mathrm{cm}$-long area from one end of the Penrose drain. Multiple small side-holes were also made at the Nelaton catheter. These two tubes were immersed in saline for lubrication. Using a long tonsil forceps, the Nelaton catheter was gently retracted to insert the Nelaton catheter within the lu-

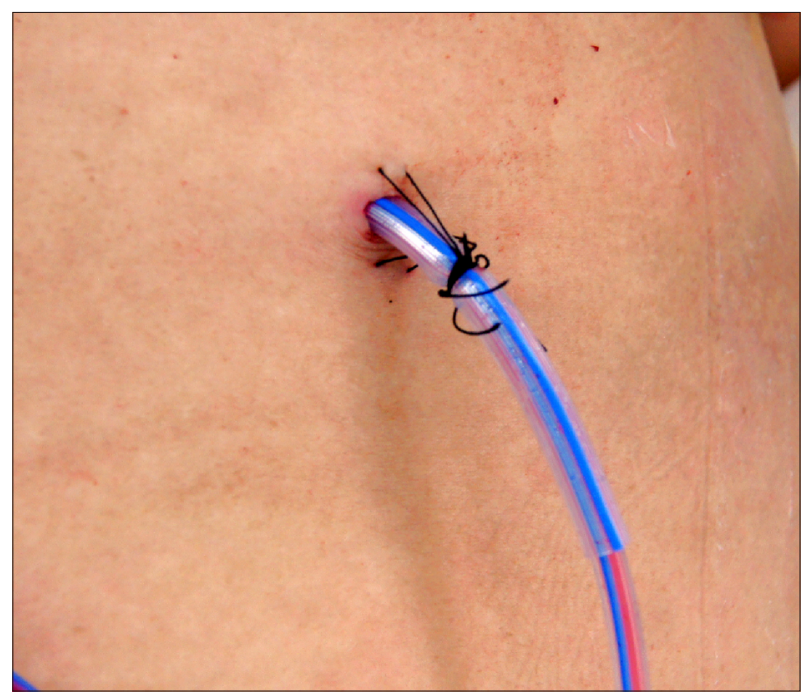

Fig. 2. Air-tight ties and skin fixation of the two-piece suction-type cigarette drain. The overlapped end of the cigarette drain is tagged and sutured at the skin and the drain tube is tied firmly twice to prevent air leak.

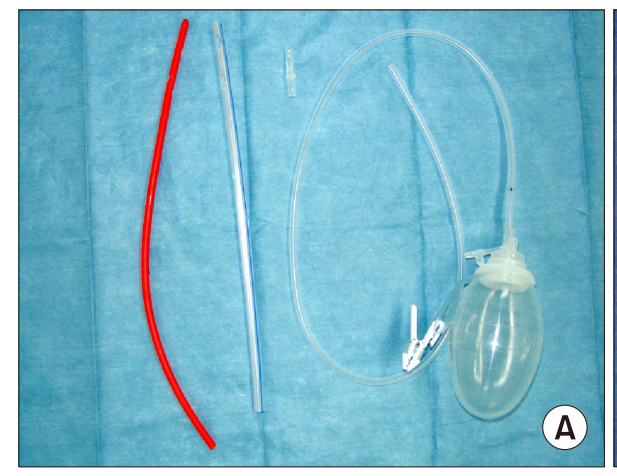

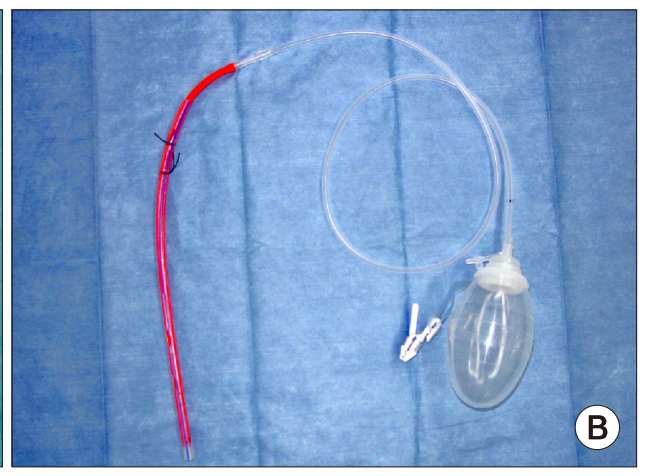

(B)
Fig. 3. Intraoperative photograph showing three-piece suction-type cigarette drain. It requires a silastic Penrose drain, a JP drain, a Nelaton tube, and a small plastic connector (A). A Nelaton catheter with multiple side-holes is placed within the lumen of a silastic Penrose drain. The overlapped end of the cigarette drain is tied firmly twice to prevent air leak (B). 

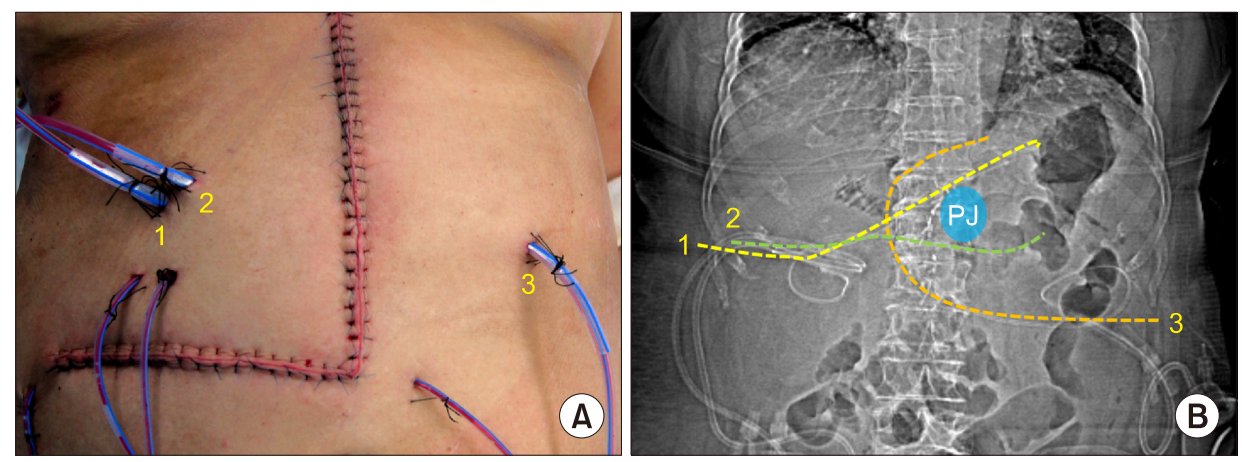

Fig. 4. Intraoperative photograph showing abdominal drain insertion in a patient treated with pylorus-preserving pancreatodudodenectomy for distal bile duct cancer. Three sets of suction-type cigarette drains (1 to 3 ) and two $3.2 \mathrm{~mm}$-sized Jackson-Pratt drains were inserted in the abdomen, with the two Jackson-Pratt drains inserted at the abdominal wound (A). The running courses of the three suction-type cigarette drains are illustrated at the simple X-ray image of the abdomen (B). PJ denotes pancreaticojejunostomy.

men of the silastic Penrose drain. The overlapped end-portion of the cigarette drain was tied firmly twice to prevent air leak (Fig. 3B).

\section{CLINICAL APPLICATION OF SUCTION-TYPE CIGARETTE DRAINS}

SCD is primarily indicated in leak-prone surgeries such as pancreatoduodenectomy and distal pancreatectomy, and extensive abdominal contamination due to intraperitoneal abscess, bowel perforation or peritonitis of other causes. Generally, we inserted three SCDs and two $3.2 \mathrm{~mm}$-sized JP drains into the abdomen of patients who underwent pancreatoduodenectomy (Fig. 4). JP drains were removed around 1 week and SCDs were usually removed after 1-3 weeks after confirmation of secured healing of the pancreaticojejunostomy. These sets of abdominal drain insertion were performed in more than 100 patients who underwent pancreatoduodenectomy, and none of them underwent repeat surgery for postoperative pancreatic fistula. Patients with extensive abdominal contamination were also managed with multiple $3.2 \mathrm{~mm}$-sized JP drains coupled with one SCD, which was located at the high-risk point of fluid collection (Fig. 5). These sets of abdominal drains were inserted in more than 30 cases of patients who underwent various abdominal surgeries. None of them underwent repeat surgery for abnormal fluid collection or abscess formation.

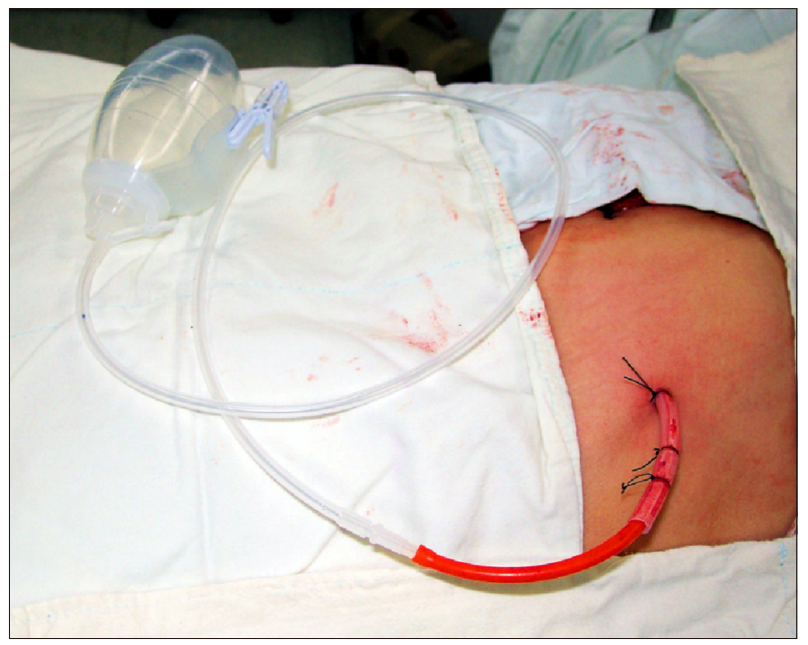

Fig. 5. Intraoperative photograph showing insertion of threepiece suction-type cigarette drain in a patient undergone contaminated upper abdominal surgery.

\section{DISCUSSION}

Effective drainage of the abnormal fluid collection is one of mainstays of treatment to prevent infection. JP drain is often not effective in case of purulent or sticky fluids. The reliability of Penrose or cigarette drains is high, but its management is painstaking. Sometimes, a colostomy bag was attached at the site of these open drains to save efforts for drain care. SCD compensates for these drawbacks of Penrose and JP drains. ${ }^{1}$

The drainage effectiveness of JP drains according to the shape of the drainage tips was reported previously. ${ }^{2}$ A laboratory model was established to compare JP drains using 3 soups to simulate body fluids containing serous, puru- 
lent, and necrotic debris. Each drain was trialed with each of the 3 fluids. The time and completeness of drainage were recorded. Under serous conditions, the round JP drain drained the cavity quicker, but left a larger residual volume of fluid. Under purulent conditions, the round JP drain was slower and drained less fluid. With debris fluid, the round JP drain was quicker with less residual fluid, whereas the flat type clogged each time. Survey results showed adequate concordance with surgeons in agreement on soup choice. The study concluded that JP drains perform differently depending on the drainage situation. Compared with the usual commercially available JP drains, our SCD carries a larger drainage tip with larger side-holes, which is more effective in drainage of purulent fluid with debris.

Closed suction drains have been routinely used in Korea for a long period, but its effectiveness was not fully assessed. A single study reported the effectiveness of drainage according to the bulbs. ${ }^{3}$ The investigators evaluated four types of popular bulb suction apparatuses to compare their maximum attainable suction, maximum volume collected, and negative pressure maintained relative to volume collected. The relative function of the Surgidyne 100cc (SD100), Jackson-Pratt 100cc (JP100), Jackson-Pratt 400cc (JP400), and HemoVac 400cc (HV400) drains were compared. The SD100 bulbs yielded the greatest negative pressure while the HV400s resulted in the least. Only the SD100s pulled at or above the purported volume. All other types obtained volumes significantly less than their described volumes: for each bulb type, the obtained volumes were statistically different. Notably, $66.7 \%$ of JP100 bulbs collected only half the purported volume. The study concluded that the use of the SD100bulb resulted in superior maximum attainable suction, maintained suction to a higher volume; they were the only bulbs tested that collected volumes at or above those purported. The HV400 bulbs demonstrated the lowest suction and volume collected. Nevertheless, when used clinically, all such drain bulbs must be emptied long before achieving the maximum vol- ume to maintain reliable suction.

Pancreatic surgery is one of the types of abdominal surgeries associated with a high risk of leak. The routine use of prophylactic abdominal drainage for pancreatic surgery has been strongly debated. Cheng et al. ${ }^{4}$ reviewed the literature to assess the benefits and harms of routine abdominal drainage after pancreatic surgery, to compare the effects of different types of surgical drains, and to evaluate the optimal time for drain removal. The study concluded that it was unclear whether routine abdominal drainage had any effect on the reduction of mortality and postoperative complications after pancreatic surgery. In case of drain insertion, low-quality evidence suggests that active drainage may reduce hospital stay after pancreatic surgery, and early removal may be superior to delayed removal for patients with a low risk of postoperative pancreatic fistula.

In conclusion, our experience suggests that SCD is highly reliable and acceptably convenient for clinical application in abdominal drainage.

\section{ORCID}

Shin Hwang: https://orcid.org/0000-0002-9045-2531

Dong-Hwan Jung: https://orcid.org/0000-0001-5984-023X

Tae-Yong Ha: https://orcid.org/0000-0001-9932-0212

\section{REFERENCES}

1. Hwang S, Ha TY, Kim JS, Cheong O, Kim KH, Lee SG. Clinical application of sution-type cigarette drain for hepatopancreatoabiliary surgery. J Korean Surg Soc 2004;67:428431.

2. Swartz AL, Azuh O, Obeid LV, Munaco AJ, Toursavadkohi S, Adams J, et al. Developing an experimental model for surgical drainage investigations: an initial report. Am J Surg 2012;203: 388-391; discussion 391.

3. Whitson BA, Richardson E, Iaizzo PA, Hess DJ. Not every bulb is a rose: a functional comparison of bulb suction devices. J Surg Res 2009;156:270-273.

4. Cheng Y, Xia J, Lai M, Cheng N, He S. Prophylactic abdominal drainage for pancreatic surgery. Cochrane Database Syst Rev 2016;10:CD010583. 